\title{
Interpreting the protective role of religiosity towards suicidal ideation using the mindsponge mechanism
}

\author{
Tam-Tri Le, Minh-Hoang Nguyen, Quan-Hoang Vuong \\ Centre for Interdisciplinary Social Research, Phenikaa University, Yen Nghia Ward, Ha Dong \\ District, Hanoi 100803, Vietnam
}

(Un-peer-reviewed version 2; January 23, 2022)

\begin{abstract}
There has been inconsistency in research on the protective effect of religions against suicide. Based on empirical findings showing that religiosity is positively associated with suicidal ideation probability among people with low social connectedness, we use the mindsponge framework of information processing to explain why religions can help protect against suicide attempts but not suicidal ideation. The aspects of information sources, subjective cost-benefit evaluation, and trust are discussed accordingly. We emphasize the role of religious leaders in conveying religious teachings which generally condemn suicide.
\end{abstract}

$$
* * * * *
$$

In 1897, Durkheim suggested that religions may have a protective effect against suicide (Durkheim, 1951). Reviews of literature on the suicide-religion relationship show that their interactions are complex, and the protective effect of religions is inconsistent (Gearing \& Alonzo, 2018; Gearing \& Lizardi, 2009; Lawrence, Oquendo, \& Stanley, 2016). Value perceptions such as moral and religious objections to suicide are suggested to be better predictors compared to religious affiliation (Dervic et al., 2011; Dervic et al., 2004). Although many studies have supported the notion that religiosity may help against suicidal attempts, findings on how religions affect suicidal ideation did not indicate the association's clear direction. There are specific cases where religions may be positively associated with suicide risk, such as through negative religious coping (Baetz \& Bowen, 2011; Lawrence, Brent, et al., 2016; Pargament, Smith, Koenig, \& Perez, 1998) or as distress responses (Taylor, Chatters, \& Joe, 2011). In particular, regarding the extant literature, Lawrence, Oquendo, et al. (2016) stated that many studies have shown that religious affiliation is protective against suicide attempts, but not suicidal ideation. In the face of this inconsistency, we attempt to investigate the underlying mechanism of suicidal ideation in relation to religiosity to explore deeper the influence of religions in terms of information processing.

The theoretical discussions in the current paper are derived from Bayesian analyses with the Hamiltonian Markov chain Monte Carlo technique (Huu \& Hoang, 2007; Huu, Vuong, \& Ngoc, 2005; Q.-H. Vuong, La, Nguyen, Ho, Ho, et al., 2020; Q.-H. Vuong, La, Nguyen, Ho, Tran, et al., 2020) using a dataset of 268 multinational students with different cultural and religious backgrounds at an international university in Japan (M.-H. Nguyen, Ho, Nguyen, \& Vuong, 2019); The results of the mentioned statistical analyses are presented in detail in the published book by 
Q.-H. Vuong, Nguyen, and Le (2021b). The main findings are summarized here. We found that although the factor of religiosity alone does not have a clear influence on suicidal ideation, religiosity is positively associated with suicidal ideation probability among those having lower social connectedness. Additionally, when facing personal and emotional difficulties, religious students are more likely to seek help from religious leaders; and those with lower social connectedness are also found to have a positive association between seeking help from religious leaders and suicidal ideation probability.

To examine the information process of suicidal ideation, including how it emerges and sustains or is ejected from the mind, we employ the mindsponge framework (Q. H. Vuong, 2016) - originally proposed by Q. H. Vuong and Napier (2015) and further developed for applying to mental health research (M.-H. Nguyen et al., 2021; M. H. Nguyen, Le, \& Meirmanov, 2019). The mindsponge framework assumes one's psychological processes as the modus operandi for receiving, evaluating, accepting/rejecting information. There are two fundamental aspects: 1) information availability (accessible sources in the current environment) and 2) information filtering using subjective costbenefit judgments based on one's current mindset. In brief, for a piece of information to exist in one's mindset (in other words, being a trusted value), it has to exist in the environment and be accessible and evaluated favorably (positive perceived net value). Such cost-benefit judgments use other trusted values within the mindset and other information from the environment as references to make connections and comparisons. Trust is a special type of trusted value that acts as a "priority pass" for certain groups of information sources or similar values. In the mindsponge framework, trust can be an energy-saving mechanism for mental activities (Le, Nguyen, \& Vuong, 2021). Mindsponge has been used effectively as a theoretical foundation for cognition-related studies in other fields such as education, environmental science, knowledge and public health management (M.-H. Nguyen \& Vuong, 2021; Q.-H. Vuong, 2021; Q.-H. Vuong et al., 2022; Q.-H. Vuong, Nguyen, \& Le, 2021a).

Based on the mindsponge framework, the aforementioned findings are explained as follows. In people with relatively higher social connectedness, they can access various sources of help-related information. Religious people are not likely to seek help from normal social sources among those with lower social connectedness due to low trust levels toward such sources. However, they still seek help from religious sources due to religious faith - a kind of trust that is not affected as much by low social connectedness. Religious teachings are a major source of mortality-related information in everyday-life contexts, including topics such as the meaning of life, the interpretation of death, and the afterlife. In people with suicidal ideation, suicide is seen as an option among other alternatives for solving the problem of suffering (M.-H. Nguyen et al., 2021). Thus, a higher reception rate of mortality-related information may increase the probability of pondering upon death and suicide. It is important to note that suicidal ideation is the ongoing evaluation of suicide's values; attempts - carrying out the act of suicide - only happen when such values reach a certain threshold (individual-based) of acceptance into the mindset. A higher intensity of evaluation does not necessarily lead to the corresponding behavior. Suicidal attempts 
can be prevented if the net perceived value of suicide is negative (in other words, when the person thinks suicide is costly in one way or another). This is why religions have a protective effect against suicidal attempts but not suicidal ideation.

During the evaluation process of suicide's values, the right interpretation of religious teachings is crucial, and it is mainly communicated through religious leaders who are trusted by their followers. While the afterlife (in whichever form according to each specific religion) may contribute to a more positive perceived value of death, suicide is generally seen as an inappropriate way to die as all major religions condemn suicide (Q.-H. Vuong et al., 2021b). For example, suicide is seen as a sin in Christianity (Retterstøl \& Ekeberg, 2009) and Islam (Okasha \& Okasha, 2009), or as a bad deed in Hinduism (Agoramoorthy \& Hsu, 2017) and Buddhism (Promta \& Thomyangkoon, 2009). Conveying the correct religious messages can greatly help people with suicidal ideation. We suggest more practical cooperation between religious leaders and mental health professionals for better insight exchange.

Mindsponge is a framework that helps with the theoretical investigation into the information process of suicidal minds through aspects of information availability, subjective cost-benefit evaluation, and trust. The framework is flexible and fits the dynamic nature of the human mind, thus making it applicable to many specific situations and research scopes. On the other hand, being a framework for information processing, mindsponge has high integrality and can help fill the gaps between other major theories on suicidality, such as the Interpersonal Theory of Suicide (Joiner, 2007; Van Orden et al., 2010), the Integrated Motivational-Volitional model (O'Connor \& Kirtley, 2018), the Fluid Vulnerability Theory of Suicide (Rudd, 2006), as well as classical works of Émile Durkheim (Durkheim, 1951). Considering the weight of the suicide issue in modern society, it is important that scientific research is properly funded (Q.-H. Vuong, 2018), and attention toward both theoretical and empirical studies on suicide should be in balance.

\section{References}

Agoramoorthy, G., \& Hsu, M. J. (2017). The Suicide Paradigm: Insights from Ancient Hindu Scriptures. Journal of Religion and Health, 56(3), 807-816. doi:10.1007/s10943-015-0178-3

Baetz, M., \& Bowen, R. (2011). Suicidal ideation, affective lability, and religion in depressed adults. Mental Health, Religion \& Culture, 14(7), 633-641. doi:10.1080/13674676.2010.504202

Dervic, K., Carballo, J. J., Baca-Garcia, E., Galfalvy, H. C., Mann, J. J., Brent, D. A., \& Oquendo, M. A. (2011). Moral or Religious Objections to Suicide May Protect Against Suicidal Behavior in Bipolar Disorder. The Journal of Clinical Psychiatry, 72(10), 1390-1396. doi:10.4088/JCP.09m05910gre

Dervic, K., Oquendo, M. A., Grunebaum, M. F., Ellis, S., Burke, A. K., \& Mann, J. J. (2004). Religious Affiliation and Suicide Attempt. American Journal of Psychiatry, 161(12), 2303-2308. doi:10.1176/appi.ajp.161.12.2303

Durkheim, E. (1951). Suicide: a study in sociology. London: Routledge.

Gearing, R. E., \& Alonzo, D. (2018). Religion and Suicide: New Findings. Journal of Religion and Health, 57(6), 2478-2499. doi:10.1007/s10943-018-0629-8 
Gearing, R. E., \& Lizardi, D. (2009). Religion and Suicide. Journal of Religion and Health, 48(3), 332-341. doi:10.1007/s10943-008-9181-2

Joiner, T. (2007). Why People Die by Suicide: Harvard University Press.

Lawrence, R. E., Brent, D., Mann, J. J., Burke, A. K., Grunebaum, M. F., Galfalvy, H. C., \& Oquendo, M. A. (2016). Religion as a risk factor for suicide attempt and suicide ideation among depressed patients. The Journal of nervous and mental disease, 204(11), 845-850. doi:10.1097/NMD.0000000000000484

Lawrence, R. E., Oquendo, M. A., \& Stanley, B. (2016). Religion and Suicide Risk: A Systematic Review. Archives of Suicide Research, 20(1), 1-21. doi:10.1080/13811118.2015.1004494

Le, T.-T., Nguyen, M.-H., \& Vuong, Q.-H. (2021). Misinformation and the Mindsponge mechanism of trust. Retrieved from https://osf.io/m9sj3

Nguyen, M. H., Le, T. T., \& Meirmanov, S. (2019). Depression, Acculturative Stress, and Social Connectedness among International University Students in Japan: A Statistical Investigation. Sustainability, 11(3), 878. doi:10.3390/su11030878

Nguyen, M.-H., \& Vuong, Q.-H. (2021). Evaluation of the Aichi Biodiversity Targets: the international collaboration trilemma in interdisciplinary research. Pacific Conservation Biology. doi:10.1071/PC21026

Nguyen, M.-H., Ho, M.-T., Nguyen, Q.-Y. T., \& Vuong, Q.-H. (2019). A Dataset of Students' Mental Health and Help-Seeking Behaviors in a Multicultural Environment. Data, 4(3), 124. doi:10.3390/data4030124

Nguyen, M.-H., Le, T.-T., Nguyen, H.-K. T., Ho, M.-T., Nguyen, H. T. T., \& Vuong, Q.-H. (2021). Alice in Suicideland: Exploring the Suicidal Ideation Mechanism through the Sense of Connectedness and Help-Seeking Behaviors. International Journal of Environmental Research and Public Health, 18(7), 3681. doi:10.3390/ijerph18073681

O'Connor, R. C., \& Kirtley, O. J. (2018). The integrated motivational-volitional model of suicidal behaviour. Philosophical Transactions of the Royal Society B: Biological Sciences, 373(1754). doi:10.1098/rstb.2017.0268

Okasha, A., \& Okasha, T. (2009). Suicide and Islam. In D. Wasserman \& C. Wasserman (Eds.), Oxford Textbook of Suicidology and Suicide Prevention (pp. 50-56): Oxford University Press.

Pargament, K. I., Smith, B. W., Koenig, H. G., \& Perez, L. (1998). Patterns of Positive and Negative Religious Coping with Major Life Stressors. Journal for the Scientific Study of Religion, 37(4), 710. doi:10.2307/1388152

Promta, S., \& Thomyangkoon, P. (2009). A Buddhist perspective on suicide. In D. Wasserman \& C. Wasserman (Eds.), Oxford Textbook of Suicidology and Suicide Prevention (pp. 28-36): Oxford University Press.

Retterst $\varnothing$ l, N., \& Ekeberg, $\varnothing$. (2009). Christianity and suicide. In D. Wasserman \& C. Wasserman (Eds.), Oxford Textbook of Suicidology and Suicide Prevention (pp. 58-62): Oxford University Press.

Rudd, M. D. (2006). Fluid Vulnerability Theory: A Cognitive Approach to Understanding the Process of Acute and Chronic Suicide Risk. In T. E. Ellis (Ed.), Cognition and suicide: Theory, research, and therapy. (pp. 355-368). Washington: American Psychological Association.

Taylor, R. J., Chatters, L. M., \& Joe, S. (2011). Religious Involvement and Suicidal Behavior Among African Americans and Black Caribbeans. Journal of Nervous \& Mental Disease, 199(7), 478-486. doi:10.1097/NMD.0b013e31822142c7

Van Huu, N., \& Hoang, V. Q. (2007). On the martingale representation theorem and on approximate hedging a contingent claim in the minimum deviation square criterion. In: Jeltsch, Li \& Sloan (Eds.) Some Topics in Industrial and Applied Mathematics (pp. 134-151). World Scientific.

Van Huu, N., Hoang, V. Q., Ngoc, T. M. (2005). Central limit theorem for functional of jump Markov processes. Vietnam Journal of Mathematics, 33(4), 443-461. 
Van Orden, K. A., Witte, T. K., Cukrowicz, K. C., Braithwaite, S., Selby, E. A., \& Joiner, T. E. (2010). The Interpersonal Theory of Suicide. Psychological review, 117(2), 575-600. doi:10.1037/a0018697

Vuong, Q. H. (2016). Global Mindset as the Integration of Emerging Socio-Cultural Values Through Mindsponge Processes : A Transition Economy Perspective. In J. Kuada (Ed.), Global Mindsets: Exploration and Perspectives (pp. 109-126). London: Routledge.

Vuong, Q. H., \& Napier, N. K. (2015). Acculturation and global mindsponge: An emerging market perspective. International Journal of Intercultural Relations, 49, 354-367. doi:10.1016/j.ijintrel.2015.06.003

Vuong, Q.-H. (2018). The (ir)rational consideration of the cost of science in transition economies. Nature Human Behaviour, 2(1), 5. doi:10.1038/s41562-017-0281-4

Vuong, Q.-H. (2021). The semiconducting principle of monetary and environmental values exchange. Economics and Business Letters, 10(3), 284-290. doi:10.17811/ebl.10.3.2021.284-290

Vuong, Q.-H., La, V.-P., Nguyen, M.-H., Ho, M.-T., Ho, M.-T., \& Mantello, P. (2020). Improving Bayesian statistics understanding in the age of Big Data with the bayesvl R package. Software Impacts, 4 , 100016. doi:10.1016/j.simpa.2020.100016

Vuong, Q.-H., La, V.-P., Nguyen, M.-H., Ho, M.-T., Tran, T., \& Ho, M.-T. (2020). Bayesian analysis for social data: A step-by-step protocol and interpretation. MethodsX, 7, 100924. doi:10.1016/j.mex.2020.100924

Vuong, Q.-H., Le, T.-T., La, V.-P., Nguyen, H. T. T., Ho, M.-T., Van Khuc, Q., \& Nguyen, M.-H. (2022). Covid-19 vaccines production and societal immunization under the serendipity-mindsponge-3D knowledge management theory and conceptual framework. Humanities and Social Sciences Communications, 9, 22. doi:10.1057/s41599-022-01034-6

Vuong, Q.-H., Nguyen, M.-H., \& Le, T.-T. (2021a). Home Scholarly Culture, Book Selection Reason, and Academic Performance: Pathways to Book Reading Interest among Secondary School Students. European Journal of Investigation in Health, Psychology and Education, 11(2), 468-495. doi:10.3390/ejihpe11020034

Vuong, Q.-H., Nguyen, M.-H., \& Le, T.-T. (2021b). A mindsponge-based investigation into the psychoreligious mechanism behind suicide attacks. Warsaw: Sciendo. 